\title{
Localised Flux Density Distribution around Different Holes on Electrical Steel: Prediction and Calculation
}

\author{
Naim Derebasi \\ Department of Physics, Bursa Uludag University, 16059 Gorukle Bursa, Turkey \\ E-mail: naim@uludag.edu.tr
}

\begin{abstract}
Localised flux density distributions are closely related to the degraded area around a cut edge or hole. Artificial neural networks (ANN) and MATLAB ${ }^{\circledR}$ Curve Fitting Toolbox ${ }^{\mathrm{TM}}$ are useful tools in prediction and analytical calculation of magnetic properties of electrical steels. A 4-node input layer, 1-node output layer model with three hidden layers, and full connectivity between nodes was developed by the ANN for prediction of localised flux density distribution. The input parameters were hole size, cutting method, induction frequency and bulk flux density while the output parameter was localised flux density due to the search coil located at the angles $0^{\circ}, 25^{\circ}, 45^{\circ}$ and $65^{\circ}$ corresponding to the centre of hole and rolling direction. The previous data obtained experimentally was used for training the proposed ANN model. Minimum correlation coefficients and RMS error for the localised flux density were found to be 0.99 and 0.09 respectively after the network was trained. The network was tested using untrained data and then minimum correlation coefficient and RMS error for localised flux density were found to be 0.98 and 0.04 respectively due to test results. A simple analytical equation has also been determined to describe the localised flux distributions. The estimation and calculation results obtained from the ANN model and analytical equations are in good agreement with experimental results previously reported.
\end{abstract}

Key words: Electrical steels, flux distribution, neural network.

\section{Special Issue of Educational Sciences}

DOI: $10.7176 / \mathrm{JSTR} / 6-06-02$

\section{Introduction}

Different cutting techniques such as mechanical, laser and abrasive waterjet cutting affect the structural features of the area near cutting in different way. The mechanical cutting induces plastic and elastic stresses near the edges of cutting line while laser cutting causes thermal stress due to rapid heating and cooling, which are harmful on magnetic properties of the material. On the other hand, in laser cutting high temperatures may cause a grain growth near the cut edge, which is beneficial on the magnetic properties. In both cases, a deterioration of the magnetic properties appears due to induced stresses $[1,2]$ and the microstructure of the material changes near the edge affecting the magnetic domain structure and domain wall motion during the magnetization process. Deterioration of magnetic properties occurs over a region up to $10 \mathrm{~mm}$ from the cut edge [3].

An alternative cutting technique, which is the abrasive waterjet cutting, was recently developed [4, 5]. Steel sheets were cut by a medium consisting of water, air and abrasive powder under high pressure.

The ANN and MATLAB ${ }^{\circledR}$ Curve Fitting Toolbox ${ }^{\mathrm{TM}}$ have been recently used for the prediction and obtaining a simple and useful analytical equation for magnetic performance [6,7]. In these materials, the flux density distribution has to be known for power loss calculation, material selection and core design. It is possible to calculate them under some assumptions; however, difficult to measure experimentally. This paper is concentrated on the prediction and calculation of localized flux density distribution around a hole in non-oriented electrical steel sheets, which was made by laser and abrasive waterjet cutting using the ANN and MATLAB ${ }^{\circledR}$ Curve Fitting Toolbox ${ }^{\mathrm{TM}}$ for optimum performance. The previous data obtained experimentally was used for training and testing the proposed ANN model [8]. 


\section{Experimental measurements}

A non-oriented $300 \mathrm{~mm}$ long, $30 \mathrm{~mm}$ wide and $0.5 \mathrm{~mm}$ thick steel sheet, which was namely N530, was cut by laser and abrasive waterjet with $10 \mathrm{~mm}$ and $20 \mathrm{~mm}$ diameter to make a hole at the center. $1 \mathrm{~mm}$ holes, which are minimizing the stress, were drilled through the sample at proper angles to wound 10turn search coils. Four search coils for each

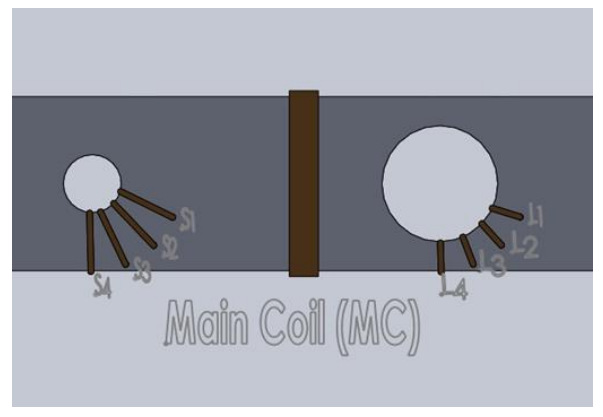

Fig.1. Search coil locations in the measurement sample.

hole with $0^{\circ}, 25^{\circ}, 45^{\circ}$ and $65^{\circ}$ angles corresponding to the center of hole were located as shown Fig. 1 [7]. A 100-turn main search coil (MC) was also wound uniformly around the sample with $0.1 \mathrm{~mm}$ enameled copper wire to detect the overall peak flux density. These samples were sinusoidally magnetized and parallel to the cut edge over the frequency 50 to $400 \mathrm{~Hz}$ for flux density level up to $0.5 \mathrm{~T}$ to avoid flux instabilities in a single-sheet tester. 10-turn $10 \mathrm{~mm}$ wide (S1-S4) and 10-turn $5 \mathrm{~mm}$ wide (L1-L4) localized search coils wound $0.10 \mathrm{~mm}$ diameter enameled copper wire were threaded through $1 \mathrm{~mm}$ diameter holes drilled through the sample and the edge of drilling hole. The voltage corresponding to magnetizing current was read across a $0.5 \mathrm{ohm}$ non-inductive shunt resistor and connected to in series to the magnetizing coil. Induced voltages from the search coils were monitored by two high precision multimeter type Agilent 3458A.

Peak magnetic flux density from the search coils was determined by the following equation;

$$
B_{m}=\frac{V_{r m s}}{4.44 N A f}
$$

where $\mathrm{N}$ is the number of turns of the search coil, $\mathrm{A}$ is the cross sectional area (product of the width of search coils and thickness of sample related to the search coil measured) and $\mathrm{f}$ is the magnetization frequency.

\section{Numerical methods}

\subsection{Artificial neural networks}

In recent years, the use of artificial neural networks has grown popularly. The reason for this is that neural networks represent a novel and modern approach, which can provide solutions to problems, and when conventional mathematics, algorithms and methodologies are unable to find a satisfactory and acceptable solution. ANNs are inspired by the human brain functionality and structure, which can be imagined as a network and comprises of densely interconnected elements called neurons [9].

The operational manner of ANNs is that when inputs are applied for the input neurons, the network performs a summation of the weighing factors and then it activates one or more specific output neurons, which are capable of providing the most suitable answer for the giving problem. The training of a supervised neural network occurs by representing typical input patterns and the corresponding expected output patterns. The weights of the connections between the neurons are modified by using an error measurement between the actual and the expected results, until the results of the network are satisfactory. For this procedure, a back propagation algorithm is usually used. It propagates the error, between the expected and actual results and backwards through the structure of the network and then it computes the weight modifications necessary to improve the actual results of the networks outputs in order to provide the most correct solution to the problem. The most popular neural network is the multi-layer perceptron, which is a feed forward network, i.e., all signals flow in a single direction from the input to the output of 
the network. It consists of neurons organized in a number of layers that can be categorized into three parts. The first part is input layer, that allows the network communicate with the environment, the second part is commonly known as the hidden part, where one or more layers of neurons exist depending on the problem's demand and generalization requirements, the third part is the out layer.

The artificial neurons are arranged in layers, wherein the output layer receives inputs from the real world and each succeeding layer receives weighted outputs from the preceding as its input resulting therefore a feed forward ANN, in which each input is fed forward to its succeeding layer, where it is treated. The outputs of the last layer constitute the outputs to the real world.

\subsection{MATLAB ${ }^{\circledR}$ Curve Fitting Toolbox}

The MATLAB ${ }^{\circledR}$ Curve Fitting Toolbox ${ }^{\mathrm{TM}}$ was used to determine a suitable analytical equation for the calculation of localized magnetic flux density $\mathrm{B}_{\mathrm{m}}$. It provides graphical tools and command-line functions for fitting curves to data [10]. The toolbox performs exploratory data analysis, pre-processes and postprocess data, compare candidate models, remove outliers and allow working an interactive environment with graphical user interface. After loading, some data a fit can be created using a fit function, specifying variables and a model type including exponential, Fourier, Gaussian, polynomial, power etc.

\section{ANN predictions}

The main problem with an ANN model has been to establish representative training data, particularly when a large number of variables are considered as in this study. A wide range previous data obtained [8] from search coils as shown Fig. 1 with varied dimensions built from $0.5 \mathrm{~mm}$ thick non-oriented steel sheet, at induction frequency from 50 to $400 \mathrm{~Hz}$ has been tested under sinusoidal wave forms. A total of 144 input vectors and magnetic flux density up to $0.5 \mathrm{~T}$ were available in the training data set. The Qnet $2000^{\circledR}$, a commercial neural network package gives the advantage of rapid network development through flexible choices of algorithms, output functions and other training parameters.

The processing ability of the network is stored in the inter-unit connection strengths or weights, obtained by a process of the adaptation to, or learning from, asset of training patterns. Models usually assume that computation is distributed over several processing units, which are interconnected and operate in parallel. Implicit knowledge is built into a neural network by training it. Some neural networks can be trained by being presented with typical input patterns and the corresponding expected output patterns. The error between the actual and expected output is used to modify the strengths, or weights, of the connections between the neurons. The back-propagation algorithm in Eq. 2 is used in this study.

$$
\delta_{k}=\sigma a_{k}\left(t_{k}^{p}-y_{k}^{p}\right)
$$

where $\mathrm{a}_{\mathrm{k}}, t_{k}^{p}, y_{k}^{p}, \delta_{\mathrm{k}}$ and $\sigma$ are $\mathrm{a}_{\mathrm{k}}$ activation neuron, $t_{k}^{p}$ target pattern neuron, $y_{k}^{p}$ output pattern neuron, $\delta_{\mathrm{k}}$ hidden layer neuron error and $\sigma$ output transfer function respectively.

The developed feed-forward neural network, which has 4 input neurons, 1 output neuron, 3 hidden layers, with 35 neurons and full connectivity between neurons was shown in Fig. 2.

Back propagation ANNs require, that all training input data have been normalized to improve the training characteristics. The input parameters were hole size, cutting method, induction frequency and overall peak flux density while the output parameter is localized peak flux density obtained from the search coils (S1, S2, S3 and S4) located around the hole. The number of hidden layers, neurons in each hidden layer and training parameters were determined through trial and error to be optimal. The network has been trained by the genetic algorithm (GA) with the hyperbolic tangent transfer function in hidden layers and output layer. The hyperbolic tangent transfer function is,

$$
\tanh x=\frac{e^{x}-e^{-x}}{e^{x}+e^{-x}}
$$

The performance of the ANN model was statistically measured by the root mean square error (RMSE) and average correlation. In calculations a HP workstation, which has an Intel ${ }^{\circledR}$ core $^{\mathrm{TM}}$ i7-720QM microprocessor, $1.6 \mathrm{GHz}, 6 \mathrm{MB} \mathrm{L} 3$ cache and $16 \mathrm{~GB}$ RAM was used. The calculation time was about more than 24 hours for each network trained. After the network was trained by the GA with 1,000,000 iteration number, the linear correlation coefficient and RMS error were found to be in the acceptable limits as shown in Table 1. Although the prediction of localized flux density was obtained both S's and L's search coils, the results are given for the S's search coils only. 


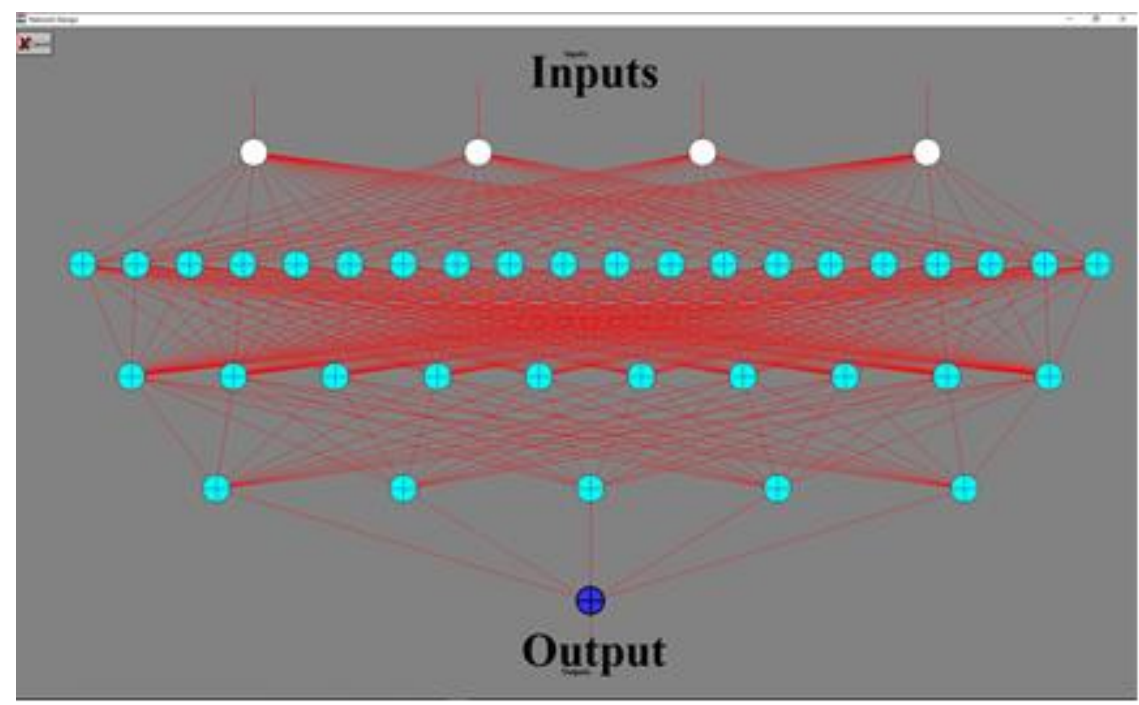

Fig. 2. Developed ANN model for prediction of localized flux densities obtained from the search coils.

When the network was tested by untrained data, minimum linear correlation coefficient and maximum RMS error were found to be $99.98 \%$ and $1.25 \%$ respectively. Table 2 also shows the percent contribution of input nodes.

The tested search coil data in the range of training data has high correlation coefficient. The ANN model was assessed from search coils located around 10, $20 \mathrm{~mm}$ diameter drilled holes, and they were outside the range of training data. From Table 2 it is clear, that the cutting method has minimum contribution for the prediction of peak flux density in the coil $\mathrm{S}_{4}$ located at $0^{\circ}$ angle while the overall peak flux density $\left(\mathrm{B}_{\mathrm{m}}\right)$ has the major contribution for the prediction of search coil flux densities.

Table 1. Properties of developed ANN model after training.

\begin{tabular}{|c|c|c|c|c|c|}
\hline \multirow{2}{*}{$\begin{array}{c}\text { Search } \\
\text { coil }\end{array}$} & \multirow{2}{*}{$\begin{array}{c}\text { Iteration } \\
\text { number }\end{array}$} & \multicolumn{2}{|c|}{ \% Linear correlation coefficient } & \multicolumn{2}{|c|}{ \% RMS error } \\
\cline { 3 - 6 } & After training & After tested & After training & After tested \\
\hline S1 & 1.000 .000 & 99.99 & 99.98 & 0.13 & 0.75 \\
\hline S2 & 1.000 .000 & 99.99 & 99.99 & 0.16 & 0.78 \\
\hline S3 & 1.000 .000 & 99.99 & 99.99 & 0.09 & 1.25 \\
\hline S4 & 1.000 .000 & 99.99 & 99.99 & 0.09 & 0.95 \\
\hline
\end{tabular}

The ANN model developed has an acceptable prediction capability for localized flux density measured by search coils within the defined training data set and good correlation with acceptable accuracy between the experimental data obtained from localized search coils and predictions. Table 3 compares the experimental data with ANN predictions for tested results.

\section{Analytical equations}

The experimental data were also analyzed by the MATLAB ${ }^{\circledR}$ curve fitting toolbox ${ }^{\mathrm{TM}}$ to obtain a simple and useful analytic equation to define the localized peak magnetic flux density $\mathrm{B}_{\mathrm{m}}$. The best analytic equation was found to be a two-term power series model. This model is applied to all data obtained from search coils around 10 and $20 \mathrm{~mm}$ diameter and laser and abrasive waterjet cut holes for better comparison.

The best curve for the localized peak flux density obtained from the search coil $\mathrm{S}_{1}$ for abrasive waterjet cutting at $50 \mathrm{~Hz}$ is defined by the analytical equation as; 
Table 2. Contribution of input nodes to localised peak magnetic flux densities in the ANN models.

\begin{tabular}{|c|c|c|c|c|}
\hline \multirow{2}{*}{ Input nodes } & \multicolumn{4}{|c|}{ Contribution \% } \\
\cline { 2 - 5 } & $\mathrm{S}_{1}$ & $\mathrm{~S}_{2}$ & $\mathrm{~S}_{3}$ & $\mathrm{~S}_{4}$ \\
\hline Hole size & 18.08 & 8.34 & 15.59 & 22.72 \\
\hline Cutting method & 18.47 & 15.79 & 14.64 & 7.92 \\
\hline $\mathrm{f}(\mathrm{Hz})$ & 27.61 & 20.73 & 15.18 & 10.38 \\
\hline $\mathrm{B}_{\mathrm{m}}(\mathrm{T})$ & 35.84 & 55.15 & 54.59 & 58.98 \\
\hline
\end{tabular}

Table 3. Comparison of the test data of search coils obtained from experiment and ANN output of peak flux density.

\begin{tabular}{|c|c|c|c|c|c|c|c|}
\hline \multicolumn{2}{|c|}{$\mathrm{B}_{\mathrm{m}}(\mathrm{T}) \mathrm{S}_{1}$} & \multicolumn{2}{c|}{$\mathrm{B}_{\mathrm{m}}(\mathrm{T}) \mathrm{S}_{2}$} & \multicolumn{2}{c|}{$\mathrm{B}_{\mathrm{m}}(\mathrm{T}) \mathrm{S}_{3}$} & \multicolumn{2}{c|}{$\mathrm{B}_{\mathrm{m}}(\mathrm{T}) \mathrm{S}_{4}$} \\
\hline Experiment & ANN & Experiment & ANN & Experiment & ANN & Experiment & ANN \\
\hline 0.627 & 0.631 & 0.521 & 0.522 & 0691 & 0.684 & 1.418 & 1.428 \\
\hline 0.390 & 0.392 & 0.330 & 0.333 & 0.485 & 0.480 & 0.934 & 0.940 \\
\hline 0.372 & 0.372 & 0.370 & 0.369 & 0.638 & 0.637 & 1.140 & 1.137 \\
\hline 0.149 & 0.150 & 0.226 & 0.224 & 0.208 & 0.212 & 0.392 & 0.388 \\
\hline 0.188 & 0.185 & 0.529 & 0.529 & 0.649 & 0.649 & 0.655 & 0.651 \\
\hline 0.177 & 0.175 & 0.484 & 0.482 & 0.631 & 0.631 & 0.659 & 0.658 \\
\hline 0.180 & 0.179 & 0.494 & 0.495 & 0.675 & 0.676 & 0.709 & 0.708 \\
\hline 0.317 & 0.316 & 0.573 & 0.570 & 0.760 & 0.758 & 0.810 & 0.809 \\
\hline 0.690 & 0.694 & 0.834 & 0.840 & 1.061 & 1.069 & 0.755 & 0.760 \\
\hline 0.175 & 0.179 & 0.397 & 0.398 & 0.729 & 0.722 & 0.498 & 0.499 \\
\hline 0.207 & 0.209 & 0.784 & 0.789 & 1.267 & 1.272 & 1.055 & 1.062 \\
\hline 0.316 & 0.308 & 0.945 & 0.953 & 1.362 & 1.369 & 1.297 & 1.300 \\
\hline 0.173 & 0.175 & 0.161 & 0.165 & 0.144 & 0.132 & 0.164 & 0.160 \\
\hline 0.291 & 0.290 & 0.480 & 0.480 & 0.438 & 0.438 & 0.414 & 0.412 \\
\hline 0.308 & 0.306 & 0.554 & 0.551 & 0.573 & 0.569 & 0.547 & 0.543 \\
\hline 0.347 & 0.348 & 0.635 & 0.633 & 0.703 & 0.701 & 0.673 & 0.678 \\
\hline
\end{tabular}

$$
B_{m}(x)=-0.15 x^{-0.44}+0.26
$$

where $x$ represents increasing steps for experimental data. The best curves for the search coils $S_{2}, S_{3}$ and $\mathrm{S}_{4}$ are also two-term power series model similar with the best curve for $\mathrm{S}_{1}$ as shown in Fig. 3a. The best analytical equation for 

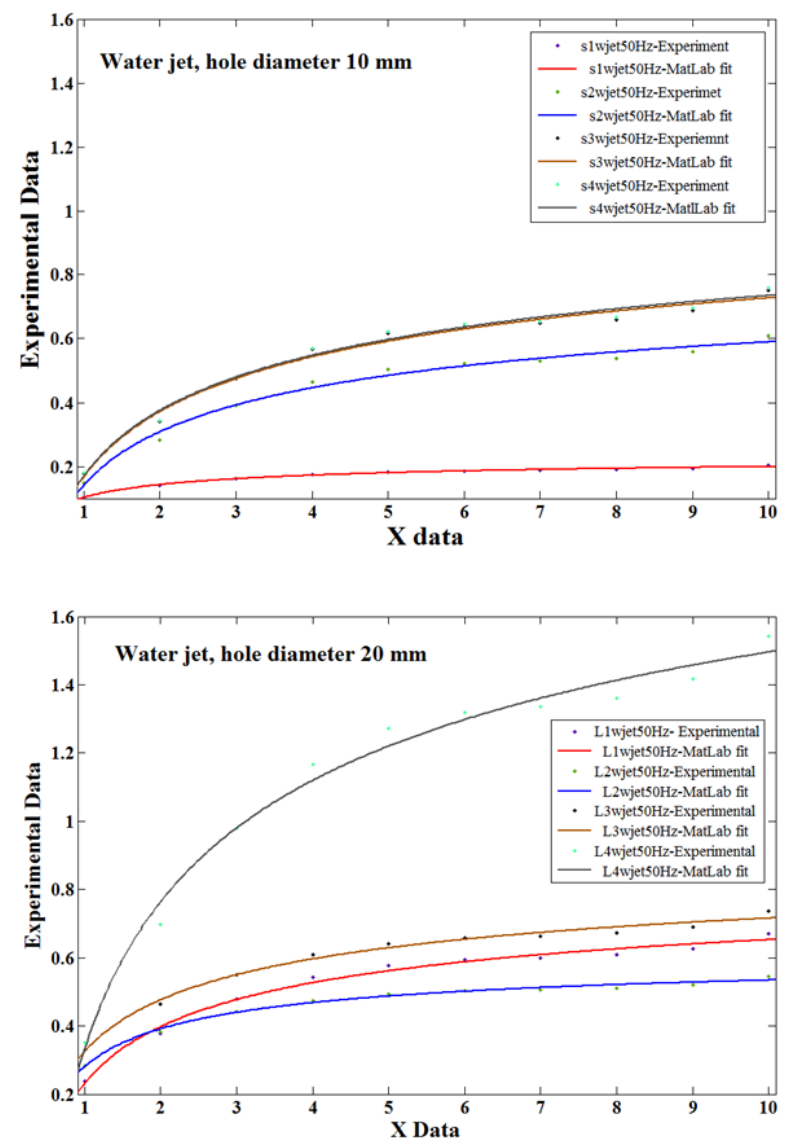

Fig. 3. a) Variation of localized peak magnetic flux density $B_{m}$ for abrasive waterjet cut $10 \mathrm{~mm}$ diameter and b) for $20 \mathrm{~mm}$ diameter holes at $50 \mathrm{~Hz}$.

$\mathrm{B}_{\mathrm{m}}$ from the search coil $\mathrm{L}_{1}$ is also defined;

$$
B_{m}(x)=0.73 x^{-0.37}+0.96
$$

as shown in Fig. $3 b$.

The best analytical equation for the search coil $\mathrm{S}_{1}$ with laser cut at $50 \mathrm{~Hz}$ is,

$$
B_{m}(x)=0.53 x^{0.33}+0.36
$$

The best curves for the search coils $S_{2}, S_{3}$ and $S_{4}$ are also similar with the best curve for $S_{1}$ as shown in Fig. 4a. The best curve for $\mathrm{B}_{\mathrm{m}}$ from the search coil $\mathrm{L}_{1}$ is; 

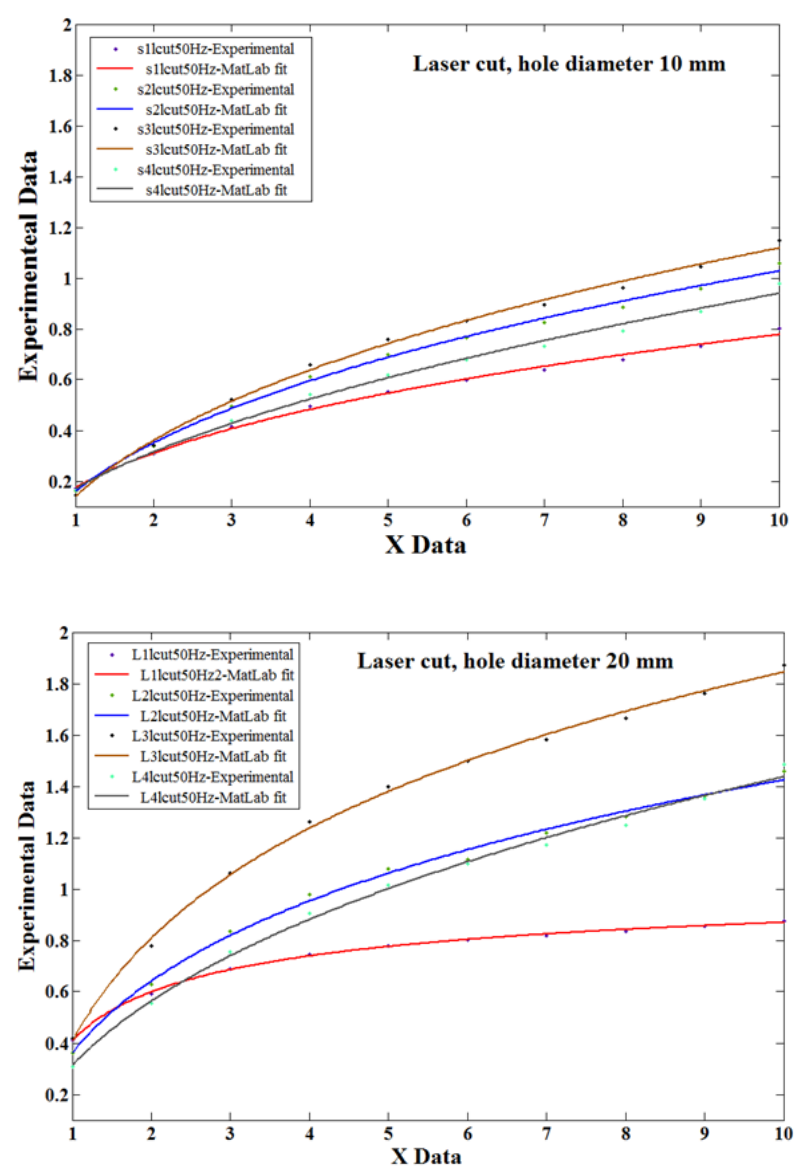

Fig. 4. a) Variation of localized peak magnetic flux density $B_{m}$ for laser cut $10 \mathrm{~mm}$ diameter and b) for $20 \mathrm{~mm}$ diameter holes at $50 \mathrm{~Hz}$.

$$
B_{m}(x)=-0.72 x^{-0.44}+1.13
$$

as shown Fig. 4b.

The R-square is the square of the correlation between the experimental values and the calculated response values. A value closer to one indicates that the model accounts for a greater proportion of variance. The RMSE is the root mean squared error or standard error. A value closer to zero indicates a fit that is more useful for calculation. Table 4 summarizes the square of the correlation and the RMSE values between experimental results and the MATLAB ${ }^{\circledR}$ curve fitting.

Minimum square correlation coefficient is $98.56 \%$ for the search coil $\mathrm{L}_{1}$ with laser cut while maximum RMS error is 4.94 for the search coil $\mathrm{L}_{4}$ with waterjet cut. Table 4 confirms that the analytic equations have an acceptable calculation capability and good correlation coefficients of acceptable accuracy within the limits of experimental and calculation values. All values obtained from Eq. (1) experimentally, prediction with ANN and calculation from MATLAB ${ }^{\circledR}$ curve fitting are in good agreement with average correlation $99 \%$ and maximum RMS error found to be $4.94 \%$. It is clear that both models, which are the ANN and analytical equations, are working well and verifying the experimental results from Eq. (1) within acceptable limits.

The ANN model developed has an acceptable prediction capability of localized flux density measured by search coils within the defined training data set and good correlation with acceptable accuracy between the experimental data and predictions. When the network was tested by untrained data, minimum linear correlation coefficient and maximum RMS error were found to be $99.98 \%$ and $1.25 \%$ respectively. The experimental data were also analyzed by the MATLAB ${ }^{\circledR}$ curve fitting toolbox ${ }^{\mathrm{TM}}$ to obtain a simple and useful analytic equation to define the localized peak magnetic flux density $\mathrm{B}_{\mathrm{m}}$. The best analytic equation was found to be a two-term power series model. Minimum square correlation coefficient was 
98.56\% while maximum RMS error was $4.94 \%$, which were within acceptable limits for experimental results.

Table 4. The square correlation and RMSE values between experimental results and MATLAB ${ }^{\circledR}$ curve fitting.

\begin{tabular}{|c|c|c|c|}
\hline \multicolumn{2}{|c|}{ Search Coil and cutting type } & Square Correlation (\%) & RMS Error (\%) \\
\hline \multirow{2}{*}{$\mathrm{S}_{1}$} & waterjet & 98.97 & 0.35 \\
\cline { 2 - 4 } & laser & 99.55 & 1.49 \\
\hline \multirow{2}{*}{$\mathrm{S}_{2}$} & waterjet & 98.62 & 1.89 \\
\cline { 2 - 4 } & laser & 99.63 & 1.93 \\
\hline \multirow{2}{*}{$\mathrm{S}_{3}$} & Waterjet & 98.57 & 2.40 \\
\cline { 2 - 4 } & laser & 99.69 & 2.01 \\
\hline \multirow{2}{*}{$\mathrm{S}_{4}$} & waterjet & 98.56 & 2.43 \\
\cline { 2 - 4 } & laser & 99.38 & 2.26 \\
\hline \multirow{2}{*}{ L1 } & waterjet & 98.85 & 1.61 \\
\cline { 2 - 4 } & laser & 99.85 & 0.63 \\
\hline \multirow{2}{*}{ L2 } & waterjet & 99.13 & 0.83 \\
\cline { 2 - 4 } & laser & 99.56 & 2.56 \\
\hline \multirow{2}{*}{ L3 } & waterjet & 98.83 & 1.51 \\
\cline { 2 - 4 } & laser & 99.80 & 2.36 \\
\hline \multirow{2}{*}{ L4 } & waterjet & 98.60 & 4.94 \\
\cline { 2 - 4 } & laser & 99.53 & 2.85 \\
\hline
\end{tabular}

\section{Acknowledgement}

The author would like to thank Mr. Cagdas Erdonmez for collecting experimental data, which were very useful and beneficial for prediction and forming an analytical equation. This research was supported by the Scientific Research Project Unit of Bursa Uludag University. The author would also like to thank for this support.

\section{References}

[1] Pulnikov A., Baudouin P. and J. Melkebeek, (2003), Induced stresses due to mechanical cutting of non-oriented electrical steels, J. Magn. Magn. Mat. 254-255, 355.

[2] Loisos G. and A. J. Moses, (2005), Effect of mechanical and Nd:YAG laser cutting on magnetic flux distribution near the cut edge of non-oriented steels, J. Mat. Proc. Tech., Vol. 161, 151.

[3] Moses A.J., Derebasi N., Loisos G. and Schoppa A., (2000), Aspects of the cut-edge effect stress on the power loss and flux density distribution in electrical steel sheets, J. Magn. Magn. Mat. 215216,690 .

[4] Schoppa A., Louis H., Pude F. and Von Rad Ch., (2003), Influence of abrasive waterjet cutting on the magnetic properties of non-oriented electrical steels, J. Magn. Magn. Mat. 254-255, 370.

[5] Shi W., Liu J. and Li C. J., (2014), Effect of cutting techniques on the structure on magnetic properties of a high-grade non-oriented electrical steel, Wuhan University of Technology-Mater. Sci. Ed. 29-6, 1246.

[6] Miti G. K., Moses A. J., Derebasi N. and Fox D., (2003), A neural network-based tool for magnetic performance prediction of toroidal cores, J. Magn. Magn. Mat. 254, 262.

32 | P a g e

www.iiste.org 
[7] Derebasi N., (2015), Effect of geometrical factors on magnetic induction distribution of toroidal cores using numerical methods, J. Supercond. Nov. Magn. 28-3, 767.

[8] Gunes, T. Derebasi N. and Erdonmez C., Localised flux density distribution around a hole in nonoriented electrical steel, IEEE Trans. Magn., 51-1, (2015) 2000204.

[9] Graupe, D. Principles of artificial neural networks, World Scientific Publishing Co. Pte. Ltd., Singapore (1997).

[10] MATLAB ${ }^{\circledR}$ Curve Fitting Toolbox ${ }^{\mathrm{TM}}$ User's Guide, R2013a. 